The latter part of the operation did not quite succeed, and he was forced to introduce a small forceps, with which the remaining portions of the capsule were removed. The inflammation following the operation was very slight; but after a few days, in consequence of the action of the aqueous humour, the lens became swollen, and by its pressure on the iris gave rise to symptoms of incipient iritis; but these were removed by an antiphlogistic treat$m \in n t$, and in four days after the operation all danger and unpleasant serisations were removed. The anterior chamber now appeared full of masses of the lens; however, these soon disappeared under the absorbing activity of the aqueous humour, and in less than the space of four weeks the pupil appeared dark and the vision was restored to a most satisfactory state.

OPERATION FOR ARTIFICIAL PUPIL.

This is an operation which very seldom succeeds, even in the hands of the most experienced and practised operators : even M. Roux, according to the testimony of M. Rognetta, fails in ten or eleven cases out of twelve.

Amongst the numerous methods of performing the operation for artificial pupil, only two are employed at the Berlin Hospital, viz., iridectomia and iridodialysis. The former was attended with results which must be considered very favourable, for in eleven cases the operation fully succeeded seven timcs, partially three times, and failed only once, while the cases treated by laceration remained in the same state as before the operation.

Lencomatous opacity of the cornea, the effect of blenorrheal ophthalmia or fiequent inflammation, was the most general indication for the formation of a new pupil, which Professor Jungken performs in the following manner: He makes an incision three lines in length close to the edge of the sclerotica: this is sufficient in all cases to produce a prolapsus of the iris, and it is by no means necessary to introduce any instrument into the anterior chamber; the prolapsed part being seized with a forceps, a portion of the iris is cut away with Cooper's small scissars so as to make as large a pupil as possible, which should be the main object of the operator. In four or five days hardly any trace of the wound made in the cornea remained, the new pupil already had assumed a larger diameter, and the vision was more or less restored.

The unsuccessful case of excision depended on the circumstance, that during the operation a spasn of the orbital muscles came on, so violent as to expel the lens, together with the iris.
Iridodialysis was performed once on a young man, twenty-three years of age, affected with partial staphyloma of the cornea ; only a narrow strip of the cornea remained clear, and the separation of the iris from the ciliary circle was effected opposite this point, though with very little hope of success. A central cataract of the lens was now for the first time discovered, and the acute inflammation which followed, left the eye nearly in the same state as before the operation.

ON THE LOCAL AND GENERAL

\section{INFLUENCE ON THE BODY, OF}

\section{INCREASED AND DIMINISHED ATMOSPHERIC PRESSURE.}

By Sir James Murra Y, M.D., T.C.D. and Ed.; M.R.C.S.Ed. \&c., Dublin.

\section{To the Editor of The LANCET.}

Sir,-Every merlical man who has been the first to propose or practise an improvement in his profession, is naturally anxious for the credit of his discovery. The process of altering the density and pressure of the atmosphere on the whole body, or on one or nore of the limbs, was subuitted by me to the profession after long reflection, but I did not think that it met with the consilleration it deserved. During the prevalence of the cholera here, all my time was engaged as a member of the Central Board of Health, and I had not a moment for personal attendance on the sick; therefore the adaptation of my theory to practice was not so well supported as I could wish. After that time, long absence in Italy, and subsequent illness, prevented my prosecuting the subject with attention, but since my return to Dublin I have renewed my endeavours to render the general and topical powers of this agent better understood, and to extend its application in aid of the treatment of many disorders. Actuated by this desire I read a paper on some of its adaptations before the Surgical Society of Ireland, and had determined to give the results of certain trials in a separate publication, but seeing the paper of Dr. Clanny in TuE LA ACET of the 7 th inst., I am now induced to submit my views to the profession through the same channel.

I am much more anxious respecting the merits of my propositions than their priority; but if Dr. Clanny entertains any doubt of the latter, I can refer him to most 
respectable medical gentlemen with whom ' was raised at this time about an inch in I have made the rarefaction and condensa- the tube, to which height it was kept as tion of air on man and other animals a nearly as possible. He complained occasubject of discussion since 1812 . The sionally of faintriess, but improved evi. application of this process to cholera was dently in the expression and colour of only a small part of $\mathrm{my}$ original proposi- countenance. After remaining sixteen sitions, as $I$ still think it applicable to minutes and a half in the bath, he was many other purposes.

Dr. Clanny has candidly awarded me the merits of the practice. I am pleased that the theory presented itself to his mind. I have no doubt that he will evince the same liberal feelings as those which actuated Dr. Orpen, when set right on this identical point by my friend Mr. Carmichael, in 1832 .

The correspondence on that occasion is so short, so convincing, and so little known (owing to the alarm which existed th at the time), that I beg leave to quote it here in reference to the late experiments of Dr. Junod, and as confirming in practice many of the anticipations which Dr. Clanny entertained in theory*:-

"In answer, Dr. Orpen sent me a very candif reply, concluding as follows: - If you wish, $\tilde{I}$ will write to the Medical Journal acknowledging that you have convinced me of the priority of your ideas; occurring first to your mind without suggestion as they did aflerwards to mine without suggestion. I am truly yours, c. E. H. OrPeN, Aug., 1832."

"This is so fair as to render further ob. sirvations unnecessary. Thefollowing case was drawn up by Mr. M'Kenna of the Cholera Hospital, by direction of $\mathrm{Dr}$. Hart. The termination was fatal, but the influence of the abstraction of air was put beyond doubt, if a proper mode of accomplishing that procedure could be easily effected, and continued for a sufficient time in the horizontal position. An artist is now preparing a bath for that purpose.

" A August 20th, 1832.-Case.-Patrick Molloy, 23, South King Street), of robust frame, entered at half past five this morning, in collapse, with blue skin, extremities cold and shrivelled. At twelve o'clock noon, was put into the air-bath. Before removal from bed he had, along with the foregoing appearances, his respiration at 36 ; pulse in the subclavian and brachial arteries 104-8. There was none to be felt on the most careful examination at the wrists; much thirst; no vomiting nor purging. After being three minutes in the bath, the respiration rose to 46 , but gradually subsided, and did not rise to above 40 afterwards; the mercury

* Printel in a work entitled the "London Med. and Surg. Jonr," but hele partly abridged by us.-Ev. L. removed at his own desire, and conveyed carefully to bed. The heat in his hody and limbs was very sensibly improved; the respiration 40 ; the pulse (which had become perceptible at the wrist) 136 ; the body not moist; the limbs cavered with a profuse perspiration, the improvement in colour and expression of countenance continuing. At a quarter of an hour after this he holds the benefits de. rived from the bath. I am bound to add, that the alterations made in the bath were the greatest improvemerits, and the machine gave us no more than unavoidable trouble.-J.W. M'Kensa. August 20th, 1832.",

Long since I proposed the abstraction of atmospheric air from the trunk of the body, I heard that some non-medical person had suggesied an apparatus for withdrawing air from the limbs, but in the latter case the principles and practice were entirely different.

The original paper sets forth my reasons for the suggestions, so plainly and explicitly, that 1 am anxious to quote it here, as well to explain the doctrine as to render the finture propositions of this paper more intelligible. Drawings are not given, because every artist is at liberty to copy any part of the apparatus, which is inuch preferable to tedious descriptions.

It is hoped that the substance of the publication of 1832 will not appear tedi. ous to the readers of THE LANCET, it being necessary as a preliminary explanation of the intended continuation of this paper.

" On the Efficacy of Diminished Atmospheric Pressure in some Cases, and of In. creased Compression or Condensation in others.

" To the Editors of the London Medical and Surgical Journal:-Gentlemen, In a work of mine on Temperature, reviewed by you in 1830, many experiments are detailed, and suggestions offered, on the influence of an altered atmosphere, both on the lungs and surface of the body. The continued investigation of these subjects since that time, both in a philosophical and medical point of view, led me to examine the effects of the common air itself, when varied in its density and pressure by art. The results of this inquiry appear so important, as to induce me to 
submit the principle for the opinion of others, before 1 could sufficiently ascertain its probable value nyself. If the re.sources of philosophy can be made available in this instance to the purposes of life, much of that delay and expense may be avoided which the imperfection of our machinery obliged me to contend with in this country.

"In the course of the experiments instituted on respiration, I observed that an animal confined in an air-tight vessel did not appear to suffer much inconvenience, if a proper supply of air were afforded for breathing by a hood adapted to the mouth and nose, while, at the same time, the air in the ressel was exhausted or rarefied by a pump to the extent of two or three pounds on the square inch. After incredible labour and expense, I succeeded in procuring a kind of bath, into which a young man was introduced, with a hood attached, leaving an open space for the eyes, mouth, and nose, and luting the margin of the opening round the cheeks and forehead by means of oiled silk or wet bladder. The pump was then connected with the bath, and the air exhausted till the mercury rose three inches in the glass-tube frtted as a guage. As might be expected the skin became instantly hot, turgid, and red, and perspiration broke out profusely; he seemed very easy, rather exhilarated, and appeared to take in large inspirations of air; the chief sensations he felt were, a drawing of the abdomen forwards, and the speedy heat of skin. rhough above two tons weight of air were removed of this man, he remained in twenty minutes, and came out very warm and wet with perspiration; the vessels of the skin were distended, and the surface red; his master, who was present, then entered the bath, and with similar results. Encouraged by the safety of these trials, I attempted to form a horizontal case or vessel, of patent air-tight cloth on hoops, but owing to the want of a good artist I could not well succeed in this attempt; others, however, may be more expert and successful.

"As I find that half a pound abstracted off the square inch produces great determination to the skin, and expansion of the chest, there is no necessity for great strength, whatever vessel or chamber may ultimately be adopted, should the principle become applicable in practice.

"The effects of a rare atmosphere are pointed out by those who have ascended very elerated situations, but in such cases the air inhaled was as light as that around the body. On the contrary, it is natural to expect different results if you place a man in a rarefied atmosphere, who is at the same time breathing air of the usual density and pressure. Abstraction of a ton or half a ton weight from the surface, permits the cutaneous ressels easily to fill, and favours their distention as it were by suction, while, at the same time, the lungs receive a column of the usual expansive force supporting the interior trunks and orsans, pressing and propelling out the blood from the centre to the circumference. In this consists the novelty and value (if any) of the principle I propose, and in this it differs from dry cupping of a part of the body, or one or more of the limbs.

"So far as respiration is concerned, the trunk resembles an elastic vessel, capable of expansion, and again of contraction by muscles and cartilages, and this contraction can take place even when the exterior pressure is taken off. If we, therefore, lift away two or three hundred weight from the circumference of the thorax and abdomen, we facilitate pulmonary expansion to that extent, by the easy descent of the diaphragm, and elevation of the walls of the chest; we then fill this vacuum with an atmosphere capable of rendering the expansion complete, without deranging the economy of the circulation.

"Whether useful or not, the means of contributing to the repletion of the lungs with, probably, double the ordinary volume of air, and, consequently, effecting a more perfect oxygenization of the blood, must present to the philosopher an agent of great power indeed. Whether this new agent shall ever become available in practice remains to be ascertained, but in theory it would seem capable of producing novel and important results, in determining revulsion from the interior to the exterior; in difficult respiration ; inward congestion; apoplexy; suppressed eruptive diseases of the skin; sudder colds, incipient fevers, or other affections depending upon the obstruction of the pores of the exterual surface; in certain female complaints; cold stages of intermittents; in short, in all partial or irregular states of the circulation, the distribution of blood to parts remote from those suffering from congestion, promises to fulfil indications in a few minutes which were formerly but very slowly effected by the lancet, or by existing medicines, and often by the reaction of Nature herself. In many diseases of joints and internal inflammations, further depletions are unsafe, from the danger of debility, though the interior inflammation requires more veresection. Can this new agent solicit the vital fluid from the centre to the circumference, and save it from being drawn off at a vein or wasting usejessly? 
In my work on 'Heat' I have pointed out the sympathy and connexion between the external skin, the medium of almost all impressions, and its continuations lining the organs of respiration and digestion, and how relieving the one instantly affects the others. A convolution of the skin turns in and lines the tubes of the lungs, presenting a surface twelve times more extended than that of the outer superficies of man. A further continuation of this cutaneous membrane lines the alimentary passages throughout all the turnings and windings, and is redoubled over their folds, to an incredible extent. All these sympathize so perfectly and suddenly, that when the exhalation on the surface is suppressed, a rapid revulsion is determined to some inward portion of this great continuous membrane. For instance, in cholera, whatever cause it is 'that poisons up the nimble spirits in the arteries,' dissolves the connexion of our elements, contracts and empties the exterior exhalents, and distends those within,--in this plague, the state of the pallid, cold, and lifeless skin, the bloodless branches and engorged trunks, the black and unoxydized blood, the constricted respiration, the compressed abdominal parietes, the suffused nervous pulp and membranes, would all seem to demand some power such as that now proposed, to restore the equilibrium of the circulation, without over stimulation : to draw out the blood not to drive it; to fill the bloodless branches, and, in proportion, empty the overloaded trunks; to generate heat from within, not to communicate it from without ; to determine from the overcharged inner organs to the empty and wrinkled skin; to impart oxygen to the blood by larger volumes of air, and, consequently, to save the brain from the fatal effects of unoxydized blood. These are some of the effects which I fondly anticipated from the resources now submitted. Whether any of the symptoms of cholera can be mitigated or retarded by this artificial process, remains yet to be ascertained. Besides the almost mechanical results expected from removing twenty or thirty hundred weight off the exterior, there are chemical and electrical considerations which may deserve attention. I observed that the air pumped off from the body whitens lime water much faster than is effected by common air, and therefore where the blood is black, drawing off carbonic acid gas from the skin may afford beneficial consequences.

"Further, independent of the abstraction of blood from the spinal column and ganglionic nerves, it appears to me that the new process may alter their electrical condition, both by insulating the body, and also by keeping it some time surrounded by a rarefied and drier atmosphere, and, consequently, modifying the electrical influence of the air on the nervous system.

"As the merits or demerits of this proposal will all present themselves to the physiologist, I need not enlarge further, but conclude by offering the following cases of cholera, drawn up by Dr. Halt, the indefatigable superintendent of the Torosisendstreet Cholera Hospital in Dublin. It is needless to observe, that no hope of ultimate recovery was entertained in either case, and that the mechanism of the bath was so imperfect as to prevent my giving a plan or drawing of it, because any good artist could soon furnish one much more simple and efficacious. Here I may observe, that it will probably be found that steam may be proved the most convenient exhauster, for every inch of steam condensed will produce a vacuum of air in the bath to the same extent. This can be effected either in the bath or by a small vessel attached by a stop-cock. Some ingenious person may also contrive a small wire frame to cover a person in bed, and draw over it a covering of air-tight cloth, furnished with an oil-leather hood for the head, or else a collar, adapted, air-tight, round the top of the sternum, clavicles, and back of the neck. Here the exhaustion of air might take place to a sufficient extent under the bed-clothes. The exhaustion should be gradual, and if carried further than the elevation of one or two inches of mercury, a common bandage might be folded round the abdomen. Where the cause of cholera affects the principle of life, it is almost hopeless to expect a cure; but when the lesion is not so destructive, some of the effects may be alleviated, and prevented from becoming new sources of progressive derangement in the rapid circle of causes and effects mutually reproducing each other in this alarming malady.

" Case 1st.-June 30th, 1832. John Keating. * * At fifty-two minutes past one o'clock p. m., no improvement having taken place, he was placed in the exhausted receiver of Dr. Murray, every part of his body being covered except the eyes, mouth, and nostrils. The pump being adjusted, the exhaustion was commenced, and continued fifteen minutes. After the first ten minutes the breathing became quicker and the breath warm. He expressed himself pleased with the effects produced, and said he was more comfortable and "snug." In fifteen minutes he complained of feeling faintish, and was removed from the apparatus. The body was now warm and moist, feet warm, but 
hands cold, and the wrists without pulse; no cramps were experienced either whilst he lay in the receiver or ofterwards. He felt a little fatigued on being removed, but was revived by the use of a little warm brandy and water. It is evident he has not been injured in the slightest degree by the process. He probably might have derived more comfort and advantage from its use, were it of such a construction as to permit the body being placed in it, and again removed, without much disturbance. JoHN HART, M.R.C.S.'

" C Case 2nd.-James Fitzpatrick, aged fifty, of broken-down constitution and drunken habits. At fifteen minutes to seven p.m., he was placed in the bath; at this time the ralial pulse was imperceptible; the carotid 100 ; respirations twentyeight in a minute; temperature of body, at axilla, 82 ; body universally bedewed with cold sweat; face and extremities blue; voice scarcely amounted to a feeble whisper. He remained in the bath twelve minutes; on being taken out, the radial pulse was distinct, 117 ; respirations, forty in a minute; body universally warm to his own sensations; by the thermometer the temperature remained the same as noted already; his voice became firm and of the natural tone. At two a.m., 5th July, his state was thus:-body and extremities warm; pulse 120 ; respiration twenty-eight; voice natural. He was given some brandy, and also carbonate of ammonia. He remained without any unfavourable change for about twelve hours, when he began to sink; the customary means were employed, but only with the success usual in such cases. He died at five p.m., on the 5th July, twenty-two hours after the trial of the experiment.

Thomas Ferrar.'

" Case 3rd.-John Thornton, ætat. forty-four, ill six hours, labouring under the usual symptoms of cholera, entered July 5th in collapse; pulse at wrist perceptible, in the carotids weak and fluttering; was placed in the exhausting bath, in which he remained about twenty minutes ; thirst continued, with great impatience for drink; seemed occasionally disposed to sleep, but with no other indication of being influenced by the removal of atmospheric pressure. On leaving the bath his energies seemed to be somewhat increased, but in a little time he fell into that comatose state which, in these cases, is commonly succeeded by death. This morning he appears a shade lighter, and is now (half past three p.m.) nothing worse.

" “ Thornton lived till eleven o"clock next day, after the above notes were taken. The three cases were beyond the reach of ? art, when at last this process was resor ted to, and though death could not be averted, the fatal termination seemed certainly to be delayed.

J. W. M'KunNa.'

"I have the honour to be,

Gentlemen, \&c. \&c. J. Murray."

The following paper, now forwarded to you for insertion in THE LANCET, was lately real by me before the surgical Society of Ireland.

Gentlemex,

Two or three interesting discussions have already taken place in the Surgical Society of Ireland, on the theory of the effects arising from dissection wounds, and $I$ hope it will not now appear out of place, if I lay before you some outlines of the principles which I think should direct our treatment on those occasions. To those who lament the loss of Dease, Shekelton, and many others, suggestions will not be considered useless on a subject which concerns the safety of the pathologist as well as that of the student of anatomy.

You are well aware, Gentlemen, that no plan hitherto devised has been found capable of preventing the progress of any subtle poison to the heart, except that which comprises some modification of "suction." You also know that during post-mortem examinations, when the hands are covered with the most offensive animal matter, suction by the mouth is seldom efficiently practised, and also that it is difficult to employ exhausting or rarefying glasses on unequal surfaces, such as those of the fingers and other parts of the hand. The consideration of these difficulties led me to contrive a metallic case for embracing the whole hand, with an adaptation to render it air-tight, either above or below the elbow, a pipe to connect it with the exhausting syringe, and another to attach it to a mercurial guage which should regulate the degree of vacuum. When the rarefaction is properly effected, it can be continued for any length of time which may be considered requisite for the protection of the patient from the effects of these wounds, as well as the effects of the bites of rabid animals, and other means of introducing deleterious matter.

This plan is certain of being effectual for spaces between the fingers, where the proper suction could not be effected by the mouth, or by any topical instrument. This mode is sure to embrace every scrateh about the parts which have been most exposed, - even such, indeed, as are not seen, felt, or suspected. It will not only act as an effectual preventive, if used by way of a precaution, but it will greatly assist the 
cure, even after the symploms have set in. Sir David Barry was able to arrest the most violent constitutional symptoms in infer ior animals by dry cupping.

Long before the discussion on these matters commenced here, I had put into the hands of your secretary, Dr. J acob, and others, a prospectus of some lectures, in which I had proposed to explain this, and similar adaptations of the principle; but as the plans require demonstration, in order to become perfectly intelligible, I shall not now trouble you with a description of them.

The value of suction in preventing the introduction of poisons into the circulation, was as well known to the ancients as is is to the moderns, and was much more frequently practised by them. The wound of Philoctetes was inflicted by one of the poisoned arrows of Hercules, and was cured by Machaon by the power of "suction" alone, as we find related in the fourth book of the Mliad. Plntarch mentions that the Psylli and Marsi were hired to attend the camp of Cato in Africa, for the purpose of sucking the bites of venomous reptiles, and that they invariably succeeded in their attempts, at the same time causing the part to swell, which proved the power of the vacuum they had produced. Hippocrates mentions the use of dry cupping to extract injurious matter from wounds :-

- Cucurbitule qua enim in usmm fabricata, Sunt ut ex carne attrabant et avellant;"

and Celsus says, that if it should so happen that the cupping-glasses are not at hand, a man must be procured to suck the part. "Homo adhibendus est qui vulnus exugat."

The experiments of Sir David Barry confirm the opinion and practice of the Greeks and Romans, and even extend so far as to prove that the application of a cupping-glass for half an hour deprives the vessels of their absorbent power during an hour or two after its removal. Modifications of the apparatus alluded to are, in my opinion, much better adapted for effecting this important object on irregular surfaces than any of the usual cupping-glasses.

Upon the subject of diminishing or abstracting atmospheric pressure, I should not omit to enforce the value of forming a vacuum immediately on opening a lumbar abscess, into which vacuum it is easy to elicit the matter by means of an elastic pipe, when such may be required. Without in this way abstracting the atmospheric air from the canal and cavity of the sac, it is almost impossible to prevent the ingress and supposed bad effects of that air.
Around an abscess in a limb you can apply circular pressure, and bring the sides of the carity into contact; but not so in cases of psoas abscess, where the bones of the pelvis, and the irregularity of the passages, prevent the application of such pressure. Besides, every motion of the lumbar and flexor muscles of the thigh, and even the act of respiration it. self, tend at intervals to make a vacuum in the sac, into which the air rushes, producing many evils, and causing the absorption of the pus and its vapour or effuvia, which cannot so readily take place if the atmospheric pressure be taken oft.

Independently of the value of this principle in the evacuation of matter, air, water, or extravasated blood, it is very advantageous in drawing out such hemorrhoidal or other tumours in or about the anus, as it may be desirable to have fully elongated for the purpose of excision, either by the knife or by ligature.

In a medical point of view, the process of rarefaction may be very important in again determining to the surface those retrocessions of eruptions, gout, rheumatism, and other translations of disease, which frequently are so injurious to the vital organs.

Rarefaction of the air is in many other ways an agent of great power and extensive adaptation; for instance, ladies in whom the development of the breasts is deficient, and who have, consequently, a paucity of milk, may have the supply required, merely by increasing the caliber of the arteries, and of course the determination to, aud retention of, the blood in those parts ; for milk is but blood altered by the vital and galvanic apparatus of the mammary glands. In cases of small contracted breasts, I have seen the abstraction of one, two, or three pounds, per inch, of pressure, expanding the vessels, augmenting their tortuosity, filling up the mam$m æ$, and producing plenty of milk in healthy subjects. By determining a similar plenitude in this manner to the uterine vessels, through the well-known sympathy of the breasts, I have frequently succeeded in causing the menses to appear after longcontinued amenorrhœa. How rational is this mode of determining the vital fluid through the mammary arteries, when compared with the mode, lately proposed, of curing obstructed menstruation by applying leeches, sinapisms, and other irritants to the breasts, and how superior to the plan just now tried in France of eliciting menstruation by sucking the nipple! The French mode only dilates that appendage; mine replenishes it, as well as the whole body of the breast. 
Having now spoken of particular applications of this principle, I shall say a few words respecting its general powers. On
the 14 th of July, 1832 , I published in the London Medical and Suryical Journal a description of an apparatus for abstract. ing part of the atmospheric pressure from the entire surface of patients. This proposal was made for cases in which it was desirable to determine the circulation from the centre to the circumference, to open the pores of the skin, induce perspiration, and cause a revulsion of any congestion from the internal organs to the exterior superficies. One of your talented members, Dr. Hart, and his associates, published the result of some trials on cholera patients, who had previously been relinquished to their certain fate at the hospital. Even then the results were sufficient to prove the great efficacy of the principle, for in all cases it rendered the cold and bloodless integurrents warm, and the capillaries permeable to the circulation. Many of you, Gentlemen, may have seen the apparatus and the publications respecting it. I now observe that in 1835 it is copied exactly by the French, and notwithstanding I had circulated the journal in Par is in 1833 on my way to Rome, yet, like most other imitators, they have misunderstood the theory, and misapplied the practice. They put the patient wholesale into the bath, pump off the air, and let the unfortunate person gasp for breath; whereas all the merit of my plan consists in furnishing the lungs with air of the usual density and pressure, while I diminish the density and pressure on the exterior parietes of the chest and whole surface of the body. At some of ny lectures I mean to show this, along with the other apparatus.

Leaving the subject of rarefaction, I shall now say a few words on the reversal of that agency, condensation. The infiuence of increased pressure, and the elasticity of the atmosphere, is a matter of great moment, and one the details of which will admit of varied extension. I am the more desirous to call your attention to this topic, without waiting for the delivery of the intended lectures, in order that $I$ may the sooner submit to your consideration the result of certain trials of the effect of atmospheric pressure. In presence of the respected Vice-President of the College of Surgeons, $\mathrm{Mr}$. White, now in the chair, I instantly reduced a very inflamed, turgid, tender, and most troublesome pro. lapsus ani, without touching the sensitive protrusion. Any of you may see the child at Larkin's, No. 32, Bridgefoot Street, in this city, and it is worth the trouble of visiting. I succeeded in a similar ex- tremely bad case last winter at Rome, after all the usual means had failed in the hands of others. The great advantage of my plan consists in this, that it empties the turigid bloodvessels, and quickly rednces the protrusion, without inflaming or irritating the rugse or folds of the mucous menbrane, and without the painful operation of introducing the fingers, paper cones, or folded cards, as formerly recommended. When the prolapsus is reduced by the pressure of air over a surface of oil, lime-water, or infusion of galls, the relaxed villons coat seldom descends again, and if it do, it returns with the greatest facility, because its veins are comparatively empty, and its morbid sensibility is subdued, or abated, by the uniform pressure.

It is almost incredible to those who do not reflect, what advantages $I$ bave seen resulting from introducing, under a cup, a wet bladder, or some other cover, an elastic compress of air upon tumours and swellings of various kinds. Among these may be reckoned chronic enlargements of the breasts and other glands, swellings in the cellular and interstitial tissues, white swellings of the joints, and distentions of the bursæ, bleeding, or fungous sores or surfaces, indurated tumours, and local congestions. Owing to my long absence, I had not sufficient opportunity to try this mode of compression in varicose veirs or aneurysmal dilatations; but I have frequently returned reducible hernia with. out pain or difficulty.

The modes of pressure recommended by Recamier, Young, and all other writers, are liable to many objections. Their compresses bear most upon the centre and sensitive apex of the tumefaction, press with great inequality, thin the parietes, and frequently hasten the degeneration of scirrhous enlargements into cancerous ulceration; but, on the contrary, the mothod here recommended presses equally upon all parts of the swelling, defends the skin from abrasion, compresses the circumference of the part, acting upon the vessels arouth the tumaur, wilere they are most capable of absorbing, propels away the contents of the veins, and prevents further untoward influx by the arteries. To persons accustomed to the manipulation of valves and machinery, these processes soon become manageable, the degrees of compression being easily regulated by a mercurial guage.

I have already mentioned that Sir David Barry and many others have proved, that the withdrawal of the air by suction or dry cupping, prevents the penetration and imbibition of poisons. I have proposed a reversal of this law, that 
is, to enforce the absorption of remedies by superadding an artificial atmospheric pressure. Warm or cold lotions, medicated infusions, gases, or vapours, may thus be introduced into the part affected, and the power of imbibition, or percolation, very much increased by well-regulated compression. In this manner solu. tions of iodine, or its vapour, sulphur, mercury, narcotics, or sedatives, can readily be introduced in local affections ur general complaints. In cases of tic douloureux, spinal disease, and sciatic ailments, I purposely detach the scarfskin, and topically introduce the appropriate remedies by pressing them in to the affected tissues, on the principle of transfusion through the membranes. On the other hand, a compression of atmospheric, or any other air, over inflamed surfaces, is well calculated to propel the overflow of blood onwards to the general circulation. I have experienced the good effects of such condensation over erysipelatous and ery thematous inflammations.

The arteries of the female breast become remarkably tortuous and distended after parturition. In cases where the mother cannot nurse, or where the child dies, this untoward circulation produces fever and irritation. It becomes, therefore, a matter of great moment to diminish the size of the bloodvessels, and lessen their activity. Cold applications, astringent lotions, and irregular compresses, have been frequently productive of serious constitutional and local injuries, but when you surround the whole mamma by a disk of proper shape, as it were, insulating the breast, and pressing only around its base, you cut off the supplies by noderate gradations, and you diminish sensibility by gentle, diffused, and elastic compression. If you prefer soothing or emollient liquids to condensed air, they can be put into the cup or disk at any temperature which is most proper or agreeable. All organs which require sudden increase of activity, and great occasional supplies, such as the womb, the stomach, the spleen, \&c., are furnished with tortuous arteries; and this twisted condition increases as the volume and active functions increase. This tortuosity may be designed by nature, not so much to impede the force of the circulation, as to detain and delay the blood in its way through the organs requiring at those periods such extra replenishment. Pressure remarkably diminishes the tortuosity of arteries which supply enlarged or inflamed glands and tumours, approximates the sides of the vessels, abates their sensibility, lessens their contents, and reduces large trunks to the diameter of minute branches. This contraction is admirably calculated to impede and expel a plenitude of blood, to diminish engorgements, and dissipate infiltrations.

Infants may die from leech-bites; the whole fanily cannot stop the hemorrhage; even the doctors have failed in extinguishing the rushlight. They all knew they could command any quantity of blood to flow by making a vacuum over the bites, but it never entered their heads that it would be just as easy to stop the orifices by making a plenum over them. Three or four strokes of the compressing piston condense the air, and instantly arrest hemorrinage. The by-standers stare, and exclaim, "How curious !" "It is so simple." "We never thought of it before." But it is not only leech-bites that conden. sation will stop, but uterine and other hemorrhages, whenever compression can be applied by means of water or other proper liquid, so as to overcome the impetus of the blood. A few inches of mercury indicate a pressure quite equal to this. This condensation you can regulate with the utmost precision, every inch of mercury indicating half a pound of pressure.

By means of well-regulated condensation of air, you can retain the bowels within the cavity of the abdomen, when its parietes have been opened or torn, and you will be able to command the flow of blood from fungous tumours and ulcerations.

Such, Mr. Editor, is the original paper read by me in the College of Surgeons of this city. In the discussion which followed, I mentioned that I did not wish to appear to prove too much by adducing additional details, but stated, that from the numerous experiments I had made on the comparatively moderate impulse of large arterial trunks, not exceeding two or three pounds of pressure, I should entertain no fear of arresting the flow of blood from any stump or limb, and thus preventing many of the accidents consequent on amputation, secure a close adaptation of the soft parts, prevent turgidity or inflammation, and effect a perfect adhesion by the first intention.

If this principle be extended to such cases, or to lacerated or penetrating wounds or torn surfaces, then the medium of water, oil, or some bland fluid, will be the best compress, condensing the air in the cup, or inverted bell, over the liquid, or in cases of round or irregular parts of the body, pressing the air into a moistened bladder or oiled silk-bag contained in the cup or cover. By these means air is prevented from entering the veins. 
$I$ forgot to mention in the paper, that in 1816 I prevented the reaction, consequent tumefaction, heat, and, probably, the sphacelation, of an extensive and dangerous laceration of my right hand (torn by glass in a chemical experiment), merely by maintaining such fluid compression orcr the bloodvessels as kept them compara. tively empty, and all the separated parts in exact apposition.

It is, probably, by diminishing the caliber of the vessels, that cold lotions, affusions, evaporating and astringent washes, were first found useful in inflammations. Perhaps it is the lacing, by the new pressure, after firing, that benefits the legs of the horse. It may be the retraction of the fibres during the shower-bath, and the consequent contraction and pressure of the skin and other tissues, that give tone to the exterior, and impulse to the interior circulation. We all remember the pressure of the plug of blood in the divided artery, and of the clot in the womb. The philosophy of a principle is sometimes much diversified in practice. Although it may be illogical to attempt to prove too much, and imprudent to provoke ridicule (the weapon only of the weak), yet I can assure your readers, that in one case where I had dissipated the cedema of a lady's legs by atmospheric pressure, her husband maintains to this day, not only that the swelling was removed, but also that the over-bulky texture of her ankles was reduced to that of a neat and sinewy joint! Whether this mode of assisting percolation be adopted or not, I can refer to the following pages of my work on the "Inhalation of Iodine, \&c.*," to prove that the subject of cutaneous imbibition was not now taken up without study and experiment.

As this communication commenced on the subject of priority, it may be proper to settle another claim. I have before me a letter from Professor Duncan, dated Edinburgh, November 15th, 18z3, from which the following is an extract: "I shall be glad to learn the results of your trials of the inhalation of iodine vapour. It is at least rational." This is exactly five years previous to Sir Charles Scudamore's thoughts upon the subject $t$, for he wrote to me in May 1830, that the idea harl first occurred to his mind two years and $a$ half prior to that date. 1 have the honour to be, Sir, your very obedient servant,

\section{Merrion Square, Dublin,}

$$
\text { James Murrar, M.D. }
$$
March 11 th, 1835.

* Vide pages 31 to $34,133,1+5$ to $1+7,157,180$, 181,301 to 303 .

\section{A S E S}

TRFATED AT THF

\section{ROSS DISPENSARY.}

\section{To the Editor of THE LANCET.}

SrR, - I am an old correspondent of TrE LANCET, and consider that perhaps the following cases may possess sufficient interest for publication. I am, Sir, Your obedient servant, John Fosbroke, M. D. Ross, Herefordshire, March 16th, 1835.

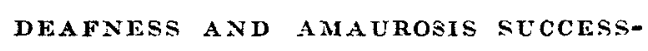

Betsy Morgan, a servant girl, xtat. 17 , of Brampton Abbots, of full and torpid habit, and heavy apprehension and manner, came under my care in the Ross Dispensary, on the 25th June 1834, at the recommendation of Edw. Pritchard, Esq. She complained of dimness of sight and of difficulty of distinguishing the forms and colours of objects, which looked black to her, but without ocular spectra; also of deafness and noises in the left ear, with deficiency of wax, occasional giddiness and headach, and suspension of the peculiar function of her sex.

The catamenia commenced at $15 x t$., but recurred irregularly at intervals of four or five months in very small quantity, and for only one day at a time. The last appearance was about five months ago. Her complaints followed "getting wet and catching colds." She was sometime ago under the care of Dr. Thomas Evans, now Physician to the Gloucester Infirmary, for darkness of sight, and received temporary relief. She has since been attended to by Mr. Thompson, one of the surgeons of the Institution, who considered the case as coming more under the province of the physician than the surgeon, and consigned her to me.

I have now had her under hand from June 25th 1834 to March 16th 1835, altogether nine months. I treated the case internally with $\mathrm{R}$ Oxym. Hyd. gr. ij ; Sp. Ather. Nit. Jij. M. Dos, coch. min. bis die.

This useful combination failed to produce the heneficial effect on the eye which frequently attends its administration at the commencement of amaurosis, and I exchanged it on the 30th July for Lugol's solution of iodine, at a dose of six drops, which she has continued till the present tine, Feb. 25th 1835, when she is taking gtt. xx, twice a day. The bowels were 